\title{
Maternal family history of hypertension attenuates neonatal pain response
}

\author{
Christopher R. France ${ }^{a}$, Anna Taddio ${ }^{b}$, Vibhuti S. Shah ${ }^{c}$, M. Gabrielle Pagéd ${ }^{d}$, Joel Katz ${ }^{\text {d,e,f,* }}$ \\ ${ }^{a}$ Department of Psychology, Ohio University, Athens, OH, USA \\ ${ }^{\mathrm{b}}$ Leslie Dan Faculty of Pharmacy, University of Toronto and Child Health Evaluative Sciences, The Hospital for Sick Children, Toronto, Ont., Canada \\ ${ }^{c}$ Department of Paediatrics, Mount Sinai Hospital and University of Toronto, Toronto, Ont., Canada \\ ${ }^{d}$ Department of Psychology. York University. Behavioural Sciences Building, 4700 Keele Street, Toronto, Ont., Canada M3J 1P3 \\ e Department of Anesthesia and Pain Management, Toronto General Hospital and Mount Sinai Hospital, Toronto, Ont., Canada \\ ${ }^{\mathrm{f}}$ Department of Anesthesia, University of Toronto, Toronto, Ont., Canada
}

\begin{abstract}
A B S T R A C T
Reduced sensitivity to naturally occurring and laboratory pain stimuli has been observed in individuals with hypertension, high-normal blood pressure, and a family history of hypertension. The present study sought to extend these findings by examining the relationship between familial history of hypertension and pain responsivity in neonates. Eighty infants had intramuscular (IM) injections of vitamin $\mathrm{K}$ performed in the delivery room within $1 \mathrm{~h}$ of birth as per institutional practice. Video recordings of the injection procedure were used by trained observers to code infant pain responses using facial grimacing and cry duration. Prior to the birth of the child, the infants' parents each completed a family blood pressure history survey and these responses were used to identify infants with and without a maternal and paternal family history of hypertension. As compared to infants without a maternal family history of hypertension, infants with a maternal family history of hypertension had significantly shorter crying times, $F(1,74)=6.96, p=.01, \eta^{2}=.086$, and marginally lower facial grimacing scores, $F(1,74)=2.68, p=.10$, $\eta^{2}=.035$, during vitamin $\mathrm{K}$ injection. The presence of attenuated responses to the IM injection in neonates with a maternal family history of hypertension provides important and novel evidence that reduced pain responding in individuals at risk for hypertension is not a learned response style, but rather may arise from prenatal or genetic influences.
\end{abstract}

\section{Introduction}

Nearly three decades of research has supported a link between blood pressure and pain perception. Specifically, reduced sensitivity to a variety of naturally occurring and laboratory pain stimuli has been observed in individuals with hypertension [16-18,22$24,39-42,47,57]$, high-normal blood pressure $[2,9,17,27,29,33,46]$, and a family history of hypertension. $[1,5,10,11,13,30,33]$. Although evidence of hypertensive hypoalgesia has also been obtained in laboratory animal studies [28,35-37,48,50,52,56], an important limitation inherent in human studies is the possibility that the hypertensive hypoalgesic effect reflects a decreased willingness to report pain rather than a higher pain threshold. This notion is supported in part by longstanding psychosomatic theories and some empirical evidence associating hypertension and risk for hypertension with affective blunting or inhibition of verbal and motoric expression of negative emotional experience

* Corresponding author. Address: Department of Psychology, York University, Behavioural Sciences Building, 4700 Keele Street, Toronto, Ont., Canada M3J 1P3. Tel.: +1 416736 2100x40557; fax: +1 4167365814 .

E-mail address: jkatz@yorku.ca (J. Katz).
$[8,25,34,55]$. While it is difficult to refute this hypothesis due to the inherently subjective nature of the pain experience, studies that have compared nociceptive flexion reflex (NFR) thresholds in offspring of hypertensive and normotensive parents provide an important insight. The NFR is a polysynaptic spinal reflex that provides an objective index of individual differences in nociceptive threshold. In the NFR paradigm, electrical stimulation is applied to the surface of the foot to determine the minimum intensity required to activate small diameter (i.e., A-delta) nociceptive fibers and a corresponding withdrawal response in the hamstring muscles of the upper leg. Comparison of NFR thresholds in healthy young adults with and without a parental history of hypertension reveals higher thresholds in offspring of hypertensive parents $[14,15,33]$, suggesting that greater nociceptive input is required to elicit pain in those at increased risk for hypertension.

The present study provides an additional, unique test of the relationship between risk for hypertension and pain perception in neonates with and without a family history of hypertension. As part of two separate investigations $[45,49]$, parental family history of hypertension was obtained before labor and delivery. Neonatal pain responses were assessed during routine IM injection of vitamin $\mathrm{K}$ (to help prevent hemorrhagic disease of the newborn). 
Because vitamin $\mathrm{K}$ injection is administered within an hour of birth, the present approach provides a rare opportunity to examine pain responses that are unlikely to be influenced by conditioning or social learning. Based on the prior evidence of attenuated pain responsivity in young adults at risk for hypertension, we hypothesized that infants with a family history of hypertension would exhibit a decreased pain responsivity as compared to infants without a family history of hypertension.

\section{Methods}

\subsection{Participants}

Data for this study were obtained from a subset of patients recruited as part of two double-blind, placebo-controlled randomized trials (RCTs) conducted in the Labor and Delivery Unit of Mount Sinai Hospital, Toronto, Canada $[45,49]$. In these studies, neonatal pain responses were assessed during routine IM injection of vitamin $\mathrm{K}$. These studies were designed to test different interventions [sucrose $24 \%$ solution [49] and amethocaine $4 \%$ gel [45]] to reduce newborn pain responses; however, when compared to the placebo control, the treatments did not significantly influence infant pain response to vitamin $\mathrm{K}$ injection. That is, neither drug proved more effective than a placebo control condition in reducing infant pain responses to vitamin $\mathrm{K}$ injection. Infants in these RCTs were included in the present study if both parents completed a survey describing their family history of hypertension.

\subsection{Procedures}

\subsubsection{Family history of hypertension}

The fathers and mothers of each infant were asked to complete a personal blood pressure history survey [32] prior to the birth of their child. Each parent completed a separate survey for their own family history. The survey inquired about age, blood pressure history (including typical blood pressure, prior diagnosis of hypertension, and antihypertensive medication prescription), and history of hypertension in first-degree relatives. Although prior studies on the relationship between pain and risk for hypertension have typically compared pain ratings in those with and without a parental history of hypertension (e.g., see France [11]), the present study used an approach to assess family history effects that was novel in two important respects. First, because of the very low incidence of hypertension in these young parents, family history of hypertension was determined on the basis of the infants' grandparents' blood pressure histories. Second, based on the evidence of specific maternal prenatal and genetic influences on risk for hypertension [7,26,54], we chose to consider the potential independent contribution of both maternal and paternal family histories.

Specifically, infants were defined as having (1) a maternal family history of hypertension if at least one of the mother's parents was reported to have had a prior diagnosis of hypertension, (2) a paternal family history of hypertension if at least one of the father's parents was reported to have had a prior diagnosis of hypertension, or (3) no family history of hypertension if neither the maternal nor the paternal grandparents were reported to have had a history of hypertension. It should be noted that paternal and maternal grandparent blood pressure histories were based on the surveys completed by the infants' father and mother, respectively, and were not independently confirmed.

\subsubsection{Vitamin $K$ injection}

The IM injection of vitamin $\mathrm{K}$ was performed in the delivery room within $1 \mathrm{~h}$ of birth as per institutional practice. The neonates were positioned supine on a resuscitation table. A digital video camera (Sony DCR-TRV530 or DCR-TRV25, Sony Electronics Inc, USA) was focused on the infant's face to record reactions to the injection. Except for exposure of the leg that was used for the injection procedure, the infants were swaddled to prevent hand movements obstructing the view of the face. A certified nurse or a physician injected the infant with $0.5 \mathrm{ml}$ of vitamin $\mathrm{K}$ (Sabex Inc, Canada) using a $1 \mathrm{ml}$ tuberculin syringe with a 25 -gauge needle $\left(5 / 8^{\prime \prime}(16-\mathrm{mm})\right.$ in depth) $[19]$. The skin of the thigh was stretched flat between the finger and the thumb and the needle was pushed down at $90^{\circ}$ angle through the skin. The injection technique was based on the guidelines recommended by the World Health Organization [53]. The injection procedure included four phases: (1) baseline, (2) cleaning the thigh, (3) injection, which included inserting the needle into the thigh, aspirating to check whether a vessel had been punctured and delivering the volume of injection, and (4) recovery.

\subsubsection{Infant pain assessment}

Neonatal facial grimacing and cry duration measures were scored from the videotaped recordings of the vitamin $\mathrm{K}$ injection. Percentage of facial grimacing was calculated on the basis of presence or absence of the three facial actions (brow bulge, eyes squeeze and deepening of the naso-labial furrow) during 2-s intervals for the first $20 \mathrm{~s}$ (or less if the phase lasted $<20 \mathrm{~s}$ ) of each procedure phase from the videotapes. The same coding procedure was used for all phases. These three facial actions were chosen for the study as they have been observed in $99 \%$ of neonates within $6 \mathrm{~s}$ of an invasive procedure (heel lance), and are believed to be the most sensitive indicators of infant pain [20]. The facial action data were collapsed for each facial action into the percentage of time the infant expressed the action (i.e., $0-100 \%$ ), and an overall score was computed by summing the percentage scores for the three facial actions and then dividing by three. The percentage of time that the infant cried during each phase was defined as the presence of audible vocalizations associated with facial grimacing. [21,58]. The pain measures were scored by a single trained research assistant (a different one for each RCT), who was not present during the videotaping, blind to the study hypotheses, and unaware of the infant's family history of hypertension. Data on the inter-rater reliability were available for facial grimacing (ICC ( $\pm 95 \% \mathrm{CI}$ ): 0.83 (0.76-0.93)) but not for cry duration.

This study was approved by the research ethics boards at Mount Sinai Hospital and the Hospital For Sick Children and the Human Participants Review Committee at York University. Parents of potentially eligible neonates were identified and approached for written consent and information concerning their family blood pressure histories prior to their child's birth.

\subsection{Statistical analysis}

To examine the effect of family history of hypertension on infant pain responses, a 2 (maternal family history of hypertension) by 2 (paternal family history of hypertension) MANOVA was conducted using difference scores (injection phase - baseline phase) for both grimacing and crying time as the dependent variables. Demographic and clinical variables were analyzed by one-way ANOVA.

\section{Results}

Eighty parent couples returned completed family history of hypertension forms that could be scored for the presence or absence of hypertension in the infants' parents and grandparents.

There were no significant differences between the infants with and without a maternal or paternal family history of hypertension 
Table 1

Mean $(95 \% \mathrm{CI}$ ) for descriptive characteristics of infants with and without a maternal family history of hypertension.

\begin{tabular}{|c|c|c|c|c|}
\hline 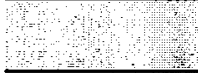 & $\begin{array}{l}\text { Positive maternal } \\
\text { family history }\end{array}$ & $\begin{array}{l}\text { Negative matemal } \\
\text { family history }\end{array}$ & p) & $\eta^{2}$ \\
\hline Kg) & 3 & & 29 & 014 \\
\hline $\begin{array}{l}\text { Gestational age } \\
\text { (weeks) }\end{array}$ & $39,24(38.88-39.59)$ & 58) & 92 & 000 \\
\hline $\begin{array}{c}\text { Mother's age } \\
\text { (years) }\end{array}$ & $34.5(32.9-36.0)$ & $329(31,5-34,2)$ & 11 & 035 \\
\hline $\begin{array}{c}\text { Father's age } \\
\text { (years) }\end{array}$ & $36.9(35.3-38.5)$ & $35.2(33.4-37.0)$ & 16 & 026 \\
\hline
\end{tabular}

in terms of birth weight, gestational age, maternal age or paternal age. Descriptive characteristics of infants as a function of maternal family history of hypertension are provided in Table 1 . There were 42 infants with and 38 without a paternal family history of hypertension, and 39 infants with and 41 without a maternal family history of hypertension.

Results of the MANOVA revealed a significant main effect of maternal family history of hypertension, $F(2,73)=3.49, p<0.05$, $\eta^{2}=.087$, but no significant effect of paternal family history of hypertension, $F(2,73)=0.91, p=0.40, \eta^{2}=.024$. The interaction effect was also non-significant. As can be seen in Table 2, follow-up ANOVAs of the maternal family history effect revealed significantly shorter crying times, $F(1,74)=6.96, p=.01, \eta^{2}=.086$, and marginally lower facial grimacing scores, $F(1,74)=2.68, p=.10, \eta^{2}=.035$, for offspring of mothers with a family history of hypertension versus those without a family history of hypertension. The maternal history effect remained significant after controlling for the treatment group to which the infants had been assigned in the original RCTs (i.e., sucrose [49] or amethocaine [45] versus placebo) and did not differ as a function of infant sex.

\section{Discussion}

The present study demonstrates a relationship between family history of hypertension and decreased pain responsivity to an IM injection in the newborn infants. Specifically, compared to infants without a maternal family history of hypertension, neonates with a maternal family history showed significantly smaller increases in crying time and marginally smaller increases in facial actions in response to the injection. These results provide novel evidence that risk for hypertension may be associated with decreased pain not as a result of social learning or environmental reinforcement, but rather as a result of undetermined prenatal or genetic influences in healthy infants with a maternal family history of hypertension.

Although this is the first study to examine hypoalgesia in infants at increased genetic risk for hypertension, similar results had previously been noted in animals. For example, spontaneously hypertensive rats (SHRs), which have been selectively bred to develop hypertension, demonstrate reduced responsiveness to nociceptive stimuli as early as the third week of life $[43,48]$. Interestingly, exaggerated endogenous opiate activity has been

Table 2

Mean (95\% $\mathrm{Cl}$ ) values for change (\% during vitamin $\mathrm{K}$ injection minus \% during preinjection baseline) in infant facial grimacing and crying time as a function of maternal and paternal family history of hypertension.

\begin{tabular}{|c|c|c|c|c|}
\hline 4 & Positive family history & Negative family history & $p$ & $n^{2}$ \\
\hline \multicolumn{5}{|c|}{ Facial grimacing } \\
\hline Mạternal & $46.4(33.2-59.6)$ & $61.4(48.7-74.0)$ & 10 & 035 \\
\hline Paternal & $574(45,1-69.8)$ & $50.3(36.9-63.8)$ & 44 & 008 \\
\hline \multicolumn{5}{|l|}{ Crying time } \\
\hline Maternal & $273(15.9-38.7)$ & $48,2(37.3-59.1)$ & 01 & 086 \\
\hline Paternal & $43.1(32.4-53.7)$ & $32.4(20.7-44,0)$ & 18 & 024 \\
\hline
\end{tabular}

identified as a mediator of hypoalgesia in SHR, since administration of the opiate-receptor antagonist naloxone normalizes nociceptive responding $[6,48,52]$. Whereas elevated plasma opioid levels have been reported in hypertensive humans $[22,31,47]$, opiate-receptor blockade studies have yielded mixed results. McCubbin and colleagues [30] demonstrated that naltrexone reversed the reduced sensitivity to cold pressor pain in those with high-normal blood pressure, and that-this effect was most pronounced in those with high-normal blood pressure and a positive family history of hypertension. In contrast, in other studies opiate-receptor blockade has failed to significantly alter nociceptive responding in individuals with hypertension [38,39], high-normal blood pressure $[29,44]$, or normotensive individuals with a family history of hypertension [12].

The demonstration of decreased pain responsivity in infants with a maternal family history of hypertension is perhaps most important in that it provides evidence of an early onset of hypoalgesia; however, this finding is unique and interesting in at least two additional respects. First, whereas prior studies have demonstrated lower pain scores as a function of hypertension or a parental history of hypertension $[1,5,10,11,13,30,33]$, this is the first time that such an effect has been reported as a function of blood pressure histories collected from grandparents. This suggests that the effect of family history of hypertension on hypoalgesic responding may be transmitted from one generation to the next, and that inheritance effects may be best estimated on the basis of a more extensive family history that goes beyond a single generation. Second, this is also the first study to examine hypoalgesic responses independently for maternal and paternal family blood pressure history. Accordingly, consistent with our finding that hypoalgesia was associated exclusively with maternal transmission of risk for hypertension, it is possible that prior findings of reduced pain responsivity in offspring of hypertensives may have been due primarily to maternal family history versus paternal family history. This notion is consistent with the emerging evidence of a maternal influence on the transmission of risk for hypertension $[7,26,54]$. Specifically, recent genetic studies demonstrate an involvement of mitochondrial DNA mutations in the development of hypertension in some families. Because mitochondrial DNA is inherited exclusively from the mother, these studies suggest a mode of inheritance of risk for hypertension that is based on a maternal transmission pattern. Accordingly, the present findings suggest that additional consideration of maternal inheritance may provide important clues in the ongoing search for potential mechanisms of hypertensive hypoalgesia in humans.

Although the present findings are intriguing, it must be noted that there are limitations to the study and methods. First, this was a pilot study including a subsample of infants, who were recruited as a part of two separate randomized controlled trials. It is, therefore, advisable that the findings be reproduced on a larger sample of infants. A larger sample size may also help address a second limitation, which relates to the fact that we observed significant group differences in infant cry times but only marginal differences in facial grimacing. Significant differences on both variables may be obtained with larger samples; however, the observed effect sizes were small, and this may be related to the challenge inherent in accurately identifying infant risk for hypertension on the basis of familial history reports. Specifically, an important limitation in all studies that use family history as a marker of hypertensive risk is the potential for both false-positive and falsenegative group assignments. False positives occur when those identified as at high risk based on family history do not go on to develop the disorder, whereas false negatives occur when those identified as at low risk based on familial history go on to develop hypertension. Because these problems occur at varying and unknown rates, the error variance associated with risk group 
assignment is likely inflated. A third and potentially related limitation is that mothers in the present sample were approximately two years younger than the fathers. Although this is a small age difference, this could increase false negatives in the maternal family history (i.e., the maternal grandparents may be younger and, therefore, may be less likely to have developed hypertension) and reduce false positives in the maternal family history as a younger diagnosis of hypertension is associated with a higher risk for the disorder in the next generation [51]. A fourth limitation is that in the present study, parental reports of hypertension in themselves or their family were not confirmed; given that prior studies have only considered a parental history of hypertension, it would be beneficial to conduct more extensive pedigree charts to provide comprehensive assessment of family history of hypertension. Finally, the absence of specific data on inter-rater reliably for сгy duration is a limitation, although coders were trained and participated in inter-rater reliability testing prior to the study. Notwithstanding these caveats, from a practical perspective hypoalgesia may serve as a valuable method of identifying those at greatest risk for hypertension among otherwise heterogeneous groups of offspring of hypertensives. Consistent with this notion, Campbell and colleagues $[3,4]$ followed a sample of young males from adolescence until early adulthood and reported that early hypoalgesia was a significant predictor of blood pressure up to eight years later, even after controlling for parental history of hypertension and initial resting blood pressure levels. Although longer follow-up studies are needed to examine the potential unique and interactive relationship between hypoalgesia and family history in the prediction of actual hypertension, hypoalgesia appears to add additional variance to the prediction of future blood pressure, and thus may serve as a behavioral marker of hypertensive risk that could be used to promote early and efficient identification of strong candidates for pharmacological and non-pharmacological prevention efforts.

\section{Acknowledgments}

The primary studies from which the data were derived were designed and conducted by V.S.S., A.T. and J.K. C.R.F. and J.K. conceived the idea and design to examine infant pain responsivity as a function of family history of hypertension. C.R.F. conducted the data analyses and drafted the initial paper, and all authors participated in the revision of the manuscript before submission. All authors have seen and given final approval of the text.

We are grateful to the parents and guardians of the participating neonates and to the nursing staff in the Labor and delivery Unit, Mount Sinai Hospital. Our special thanks to the research assistants Amanda Baker, Jessica Singh, graduate students Rebecca Hancock, Ryan Smith, and respiratory therapist Laura Noble who assisted in recruitment and videotaping the study neonates, to Erwin Darra and Lucia Taddio for aspects of videotape analysis, and to Eileen Halket for data entry.

The project was funded in part by the "Striving for Excellence Fund", Mount Sinai Hospital awarded to V.S.S., Canadian Institutes of Health Research (CIHR) Grant 63143 to A.T., the Canadian Foundation for Innovation, Ontario Innovation Trust, a CIHR Canada Research Chair in Health Psychology to J.K. and CIHR New Investigator Award to A.T. C.R.F. is funded through the National Institutes of Health NIH R01 HL077438 and NIH R01 HD045512. The authors have no conflicts of interest.

\section{References}

[1] al'Absi M, Buchanan T, Lovallo WR. Pain perception and cardiovascular responses in men with positive parental history for hypertension. Psychophysiology 1996;33:655-61.

[2] Bruehl S, Carlson CR, McCubbin JA. The relationship between pain sensitivity and blood pressure in normotensives. Pain 1992;48:463-7.
[3] Campbell TS, Ditto B, Seguin JR, Assaad JM, Pihl RO, Nagin D, et al. A longitudinal study of pain sensitivity and blood pressure in adolescent boys: results from a 5-year follow-up. Health Psychol 2002;21:594-600.

[4] Campbell-TS, Ditto B, Seguin JR, Sinray S, Tremblay RE. Adolescent pain sensitivity is associated with cardiac autonomic function and blood pressure over 8 years. Hypertension 2003;41:1228-33.

[5] Cook DB, Jackson EM, O'Connor PJ, Dishman RK. Muscle pain during exercise in normotensive African-American women: effect of parental hypertension history. J Pain 2004;5:111-8.

[6] Delbarre B, Casset-Senon D, Delbarre G, Sestillange P, Christin O. Naloxone effects on blood pressure, analgesia and diuresis in spontaneous hypertensive and normotensive rats. Neurosci Lett 1982;30:167-72.

[7] Delles C. Mitochondria, maternal transmission and hypertension. J Hypertens 2007:25:2001-3.

[8] Engel BT. An historical and critical review of the articles on blood pressure published in psychosomatic medicine between 1939 and 1997. Psychosom Med 1998;60:682-96.

[9] Fillingim RB, Maixner W. The influence of resting blood pressure and gender on pain responses, Psychosom Med 1996;58:326-32.

[10] France C, Ditto B, Adler P. Pain sensitivity in offspring of hypertensives at rest and during baroreflex stimulation. J Behav Med 1991;14:513-25.

[11] France CR. Decreased pain perception and risk for hypertension: considering a common physiological mechanism. Psychophysiology 1999;36:683-92.

[12] France CR, al'absi M, Ring C, France JL, Brose J, Spaeth D, et al. Assessment of opiate modulation of pain and nociceptive responding in young adults with a parental history of hypertension. Biol Psychol 2005;70:168-74.

[13] France CR, Ditto B. Risk for high blood pressure and decreased pain perception. Curr Direct Psychol Sci 1996;5:120-5.

[14] France CR, Froese SA, Stewart JC. Altered central nervous system processing of noxious stimuli contributes to decreased nociceptive responding in individuals at risk for hypertension. Pain 2002;98:101-8.

[15] France CR, Suchowiecki S. Assessing supraspinal modulation of pain perception in individuals at risk for hypertension. Psychophysiology 2001;38:107-13.

[16] Ghione S. Hypertension-associated hypalgesia. Evidence in experimental animals and humans, pathophysiological mechanisms, and potential clinical consequences. Hypertension 1996;28:494-504.

[17] Ghione S, Rosa C, Mezzasalma L, Panattoni E. Arterial hypertension is associated with hypalgesia in humans. Hypertension 1988;12:491-7.

[18] Ghione S, Rosa C, Panattoni E, Nuti M, Mezzasalma L, Giuliano G, et al. Comparison of sensory and pain threshold in tooth pulp stimulation in normotensive man and essential hypertension. J Hypertens 1985:S113-5.

[19] Groswasser J, Kahn A, Bouche B, Hanquinet S, Perlmuter N, Hessel L, et al. Needle length and injection technique for efficient intramuscular vaccine delivery in infants and children evaluated through an ultrasonographic determination of subcutaneous and muscle layer thickness. Pediatrics 1997;100:400-3.

[20] Grunau RV, Craig KD. Pain expression in neonates: facial action and cry. Pain 1987;28:395-410.

[21] Grunau RV, Johnston CC, Craig KD. Neonatal facial and cry responses to invasive and non-invasive procedures. Pain 1990;42:295-305.

[22] Guasti L, Cattaneo R, Daneri A, Bianchi L, Gaudio G, Regazzi MB, et al. Endogenous beta-endorphins in hypertension: correlation with 24-hour ambulatory blood pressure. J Am Coll Cardiol 1996:28:1243-8.

[23] Guasti L, Cattaneo R, Rinaldi O, Rossi MG, Bianchi L, Gaudio G, et al. Twentyfour-hour noninvasive blood pressure monitoring and pain perception. Hypertension 1995;25:1301-5.

[24] Guasti L, Gaudio G, Zanotta D, Grimoldi P, Petrozzino MR, Tanzi F, et al. Relationship between a genetic predisposition to hypertension, blood pressure levels and pain sensitivity. Pain 1999;82:311-7.

[25] Jorgensen RS, Johnson BT, Kolodziej ME, Schreer GE. Elevated blood pressure and personality: a meta-analytic review. Psychol Bull 1996;120:293-320.

[26] Li Z, Liu Y, Yang L, Wang S, Guan MX. Maternally inherited hypertension is associated with the mitochondrial tRNA(Ile) A4295G mutation in a Chinese family. Biochem Biophys Res Commun 2008;367:906-11.

[27] Maixner W, Fillingim R, Kincaid S, Sigurdsson A, Harris MB. Relationship between pain sensitivity and resting arterial blood pressure in patients with painful temporomandibular disorders. Psychosom Med 1997;59:503-11

[28] Maixner W, Touw KB, Brody MJ, Gebhart GF, Long JP. Factors influencing the altered pain perception in the spontaneously hypertensive rat. Brain Res 1982;237:137-45.

[29] McCubbin JA, Bruehl S. Do endogenous opioids mediate the relationship between blood pressure and pain sensitivity in normotensives? Pain 1994;57:63-7.

[30] McCubbin JA, Helfer SG, Switzer 3rd FS, Galloway C, Griffith WV. Opioid analgesia in persons at risk for hypertension. Psychosom Med 2006;68:116-20.

[31] McNeilly $M$, Zeichner A. Neuropeptide and cardiovascular responses to intravenous catheterization in normotensive and hypertensive blacks and whites. Health Psychol 1989;8:487-501.

[32] Page GD, France CR. Identifying hypertension using the Ohio Blood Pressure History Survey. Military Med 2001;166:233-6.

[33] Page GD, France CR. Objective evidence of decreased pain perception in normotensives at risk for hypertension. Pain 1997;73:173-80. 
[34] Pury CL, McCubbin JA, Helfer SG, Galloway C, McMullen L]. Elevated resting blood pressure and dampened emotional response. Psychosom Med 2004;66:583-7.

[35] Randich A, Maixner W. Interactions between cardiovascular and pain regulatory systems. Neurosci Biobehav Rev 1984;8:343-67.

[36] Randich A, Maixner $W$. The role of sinoaortic and cardiopulmonary baroreceptor reflex arcs in nociception and stress-induced analgesia. Ann NY Acad Sci 1986;467:385-401.

[37] Randich A, Robertson JD. Spinal nociceptive transmission in the spontaneously hypertensive and Wistar-Kyoto normotensive rat. Pain 1994;58:169-83.

[38] Ring C, France CR, al'Absi M, Beesley L, Edwards L, Mclntyre D, et al. Effects of opioid blockade with naltrexone and distraction on cold and ischemic pain in hypertension. J Behav Med 2007;30:59-68.

[39] Ring C, France CR, Al'absi M, Edwards L, McIntyre D, Carroll D, et al. Effects of naltrexone on electrocutaneous pain in patients with hypertension compared to normotensive individuals. Biol Psychol 2008;77:191-6.

[40] Rosa C, Ghione S. Effect of ketanserin on pain perception in arterial hypertension. Cardiovasc Drugs Ther 1990;4:133-5.

[41] Rosa C, Ghione S, Panattoni E, Mezzasalma L, Giuliano G. Comparison of pain perception in normotensives and borderline hypertensives by means of a tooth pulp-stimulation test. J Cardiovasc Pharmacol 1986;8:S125-7.

[42] Rosa C, Vignocchi G, Panattoni E, Rossi B, Ghione S. Relationship between increased blood pressure and hypoalgesia: additional evidence for the existence of an abnormality of pain perception in arterial hypertension in humans. J Hum Hypertens 1994;8:119-26.

[43] Saavedra JM. Naloxone reversible decrease in pain sensitivity in young and adult spontaneously hypertensive rats. Brain Res 1981;209:245-9.

[44] Schobel HP, Handwerker HO, Schmieder RE, Heusser K, Dominiak P, Luft FC, et al. Effects of naloxone on hemodynamic and sympathetic nerve responses to pain in normotensive vs. borderline hypertensive men. J Auton Nerv Syst 1998;69:49-55.

[45] Shah VS, Taddio A, Hancock R, Shah P, OhIsson A. Topical amethocaine gel 4\% for intramuscular injection in term neonates: a double-blind, placebocontrolled, randomized trial. Clin Ther 2008;30:166-74.

[46] Sheffield D, Krittayaphong R, Go BM, Christy CG, Biles PL, Sheps DS, et al. The relationship between resting systolic blood pressure and cutaneous pain perception in cardiac patients with angina pectoris and controls. Pain 1997;71:249-55.

[47] Sheps DS, Bragdon EE, Gray 3rd TF, Ballenger M, Usedom JE, Maixner W, et al. Relation between systemic hypertension and pain perception. Am J Cardiol 1992:70:3F-5F

[48] Sitsen JM, de Jong W. Hypoalgesia in genetically hypertensive rats (SHR) is absent in rats with experimental hypertension. Hypertension 1983;5:185-90.

[49] Taddio A, Shah V, Hancock R, Smith RW, Stephens D, Atenafu E, et al Effectiveness of sucrose analgesia in newborns undergoing painful medical procedures. Cmaj 2008;179:37-43.

[50] Tsai CF, Lin MT. Pain sensitivity, thermal capability, and brain monoamine turnover in hypertensive rats. Am J Physiol 1987;253:R910-6.

[51] Wang NY, Young JH, Meoni LA, Ford DE, Erlinger TP, Klag M], et al, Blood pressure change and risk of hypertension associated with parental hypertension: the Johns Hopkins Precursors Study. Arch Int Med 2008;168:643-8.

[52] Wendel OT, Bennett B. The occurrence of analgesia in an animal model of hypertension. Life Sci 1981;29:515-21.

[53] World Health Organization. Immunization in practice (a guide for health workers who give vaccines).

[54] Yang Q, Kim SK, Sun F, Cui J, Larson MG, Vasan RS, et al. Maternal influence on blood pressure suggests involvement of mitochondrial DNA in the pathogenesis of hypertension: the Framingham Heart Study. J Hypertens 2007;25:2067-73.

[55] Younger JW, Lawler-Row KA, Moe KA, Kratz AL, Keenum AJ. Effects of naltrexone on repressive coping and disclosure of emotional material: a test of the opioid-peptide hypothesis of repression and hypertension. Psychosom Med 2006;68:734-41.

[56] Zamir N, Maixner $W$. The relationship between cardiovascular and pain regulatory systems. Ann NY Acad Sci 1986;467:371-84.

[57] Zamir $N$, Shuber E. Altered pain perception in hypertensive humans. Brain Res 1980;201:471-4.

[58] Zeifman D, Delaney S, Blass EM. Sweet taste, looking and calm in 2- and 4week-old infants: the eyes have it. Dev Psychol 1996;32:1090-9. 